Pacific Journal of Mathematics

REPRESENTATIONS OF HOMOLOGY 3-SPHERES 


\title{
REPRESENTATIONS OF HOMOLOGY 3-SPHERES
}

\author{
HUGH M. HILDEN
}

\begin{abstract}
Every homology 3-sphere can be represented as a framed link, in the sense of Kirby, of the following special type. There are $k$ disjoint embeddings in $S^{3}$ of a genus one surface with two boundary components. The link is the $2 k$ boundary components. If $q$ is the linking number of the $i$ th pair, then one of the components has framing $q+1$, the other $q-1$.
\end{abstract}

1. Introduction. A very useful and fairly recent method for studying 3-manifolds, in particular for the study of examples, is R. Kirby's "Calculus of framed links" (see [8], [7]). Another, older, method is through the mapping class group of a surface via Heegaard splittings (see [1], [4]). Through the work of Birman [2], Powell [9], and Johnson [6], a geometrically appealing (but infinite) set of generators has been found for group of homeomorphisms of an orientable surface, up to isotopy, that induce the identity on homology. It is the purpose of this paper to use this set of generators to obtain a representation theorem for all homology 3 -spheres as special framed links. It turns out that the formula for the $\mu$-invariant of homology 3 -spheres represented this way is particularly simple.

2. Notation and conventions. Throughout the paper $X_{g}$ will be a 3-dimensional genus $g$ handlebody, $T_{g}$ its boundary, $X_{g}^{\prime}$ another such handlebody, and $i$ a PL homeomorphism from $T_{g}$ to $\partial X_{g}^{\prime}$ so that $X_{g} \cup X_{g}^{\prime}$ defines a Heegaard splitting of $S^{3}$. Also, $A$ will be an "annulus with a handle"; that is an oriented genus one surface with two boundary components. The boundary components derive orientations from the orientation of $A$ and will be denoted $a$ and $b$. We assume the reader is familiar with such concepts as "characteristic surface", "intersection matrix", "index", and " $\mu$-invariant" as they apply to three and four manifolds. These terms are defined in [8].

3. Several propositions. In this section we state several results needed for the proof of the main theorem.

Proposition 1. Let $H^{3}$ be a genus g homology 3-sphere. There is a homeomorphism $\phi$ of $T_{g}$ such that $\phi$ induces the identity on the homology of $T_{g}$ and $H^{3}=X_{g} \bigcup_{i \phi} X_{g}^{\prime}$. 
Proof. This is proven by Joan Birman in [3].

Let $f: A \rightarrow T_{g}$ be an embedding. If we do Dehn twists in opposite directions about the boundary curves of $f(A)$, it is not difficult to see that this homeomorphism induces the identity on the homology of $T_{g}$. We shall call such a homeomorphism "special" for awhile.

Proposition 2. Let $\phi$ be a homeomorphism of $T_{g}$ that induces the identity on homology. Then $\phi$ is isotopic to a finite product of "special" homeomorphisms.

Proof. This follows directly from results of Dennis Johnson ([6]), Jerome Powell ([9]), and Joan Birman ([2]).

Proposition 3. Let $M^{3}=X_{g} \bigcup_{\alpha_{n} \cdots \alpha_{1} i} X_{g}^{\prime}$ where $\alpha_{j}$ is a homeomorphism of $T_{g}, 1 \leqq j \leqq n$. Then also

$$
M^{3}=X_{g} \bigcup_{i d} T_{g} \times[0,1] \bigcup_{\hat{\alpha}_{1}} T_{g} \times[1,2] \bigcup_{\hat{\alpha}_{2}}^{\bigcup} \cdots \bigcup_{\hat{\alpha}_{n}} T_{g} \times[n, n+1] \bigcup_{i} X_{g}^{\prime},
$$

where $\hat{\alpha}_{j}(x, j)=\left(\alpha_{j}(x), j\right)$.

Proof. We can present $M^{3}$ as

$$
M^{3}=X_{g} \bigcup_{i d} T_{g} \times[0,1] \bigcup_{i d} T_{g} \times[1,2] \cup \cdots \bigcup_{i d} T_{g} \times[n, n+1] \bigcup_{\alpha_{n} \cdots \alpha_{1} i} X_{g}^{\prime}
$$

and then explicitly define a homeomorphism as below.

$$
\begin{aligned}
& X_{g} \bigcup_{i d} T_{g} \times[0,1] \bigcup_{\hat{\alpha}_{1}} T_{g} \times[0,2] \bigcup_{\hat{\alpha}_{2}} \cdots \bigcup_{\hat{\alpha}_{n}} T_{g} \times[n, n+1] \bigcup_{i} X_{g}^{\prime} \\
& \uparrow i d \quad \uparrow i d \alpha_{1} \times i d \quad \uparrow \alpha_{2} \alpha_{1} \times i d \cdots \uparrow \alpha_{n} \cdots \alpha_{1} \times i d \quad \uparrow i d \\
& X_{g} \bigcup_{i d} T_{g} \times[0,1] \bigcup_{i d} T_{g} \times[0,2] \cup \cdots \bigcup_{i d} T_{g} \times[n, n+1] \bigcup_{\alpha_{n} \cdots \alpha_{1} i} X_{g}^{\prime}
\end{aligned}
$$

4. Statement and proof of the main theorem.

MaIN THeOREM. Let $f_{i}, 1 \leqq i \leqq n$ be a set of smooth embeddings of $A$ in $S^{3}$ with pairwise disjoint images. Let $q_{i}$ be the linking number of $f_{i}(a)$ and $f_{i}(b)$. Frame the link $\left\{f_{1}(a), f_{1}(b) ; f_{2}(a), f_{2}(b), \cdots\right.$, $\left.f_{n}(b)\right\}$ with framing numbers $\left\{-q_{1}+1,-q_{1}-1 ;-q_{2}+1, \cdots,-q_{n}-1\right\}$ respectively and let $M^{3}$ be the 3-manifold constructed from this framed link using the Kirby calculus ([8]).

Then $M^{3}$ is a homology 3 -sphere and every homology 3-sphere can be constructed this way. The $\mu$-invariant of $M^{3}$ (as an integer 
$\bmod 2)$ is the mod 2 sum of the $\operatorname{Arf}$ invariants of the surfaces $f_{i}(A)$ that have $q_{i}$ even.

Proof. We begin by showing that an arbitrary homology 3 -sphere $H^{3}$ has this type of representation. It follows directly from Propositions 1, 2 and 3 that

$$
H^{3}=X_{g} \bigcup_{i d} T_{g} \times[0,1] \bigcup_{\hat{\alpha}_{1}} T_{g} \times[1,2] \bigcup_{\hat{\alpha}_{2}} \cdots \bigcup_{\hat{\alpha}_{n}} T_{g} \times[n, n+1] \bigcup_{i} X_{g}^{\prime}
$$

where $S^{3}=X_{g} \bigcup_{i d} T_{g} \times[0,1] \bigcup_{i d} T_{g} \times[1,2] \cup \cdots \bigcup_{i d} T_{g} \times[n, n+1] \bigcup_{i} X_{g}^{\prime}$ and $\hat{\alpha}_{j}: T_{g} \times\{j\} \rightarrow T_{g} \times\{j\}$ is "special". Thus there are embeddings $f_{j}: A \rightarrow T_{g} \times\{j\}, 1 \leqq j \leqq n$ and $\hat{\alpha}_{j}$ consists of simultaneous Dehn twists in opposite directions about the bounding curves of $f_{j}(A)$ in the surface $T_{g} \times\{j\}$. Denote $f_{j}(a)$ and $f_{j}(b)$ by $k_{1}$ and $k_{2}$ respectively.

For $i=1,2$ let $U_{i}$ be a tubular neighborhood of $k_{i}$, let $m_{i}$ be a meridian, let $l_{i}$ be a longitude, and let $s_{i}=f_{j}(A) \cap \partial U_{i}$. Assume $m_{i}, l_{i}$, and $s_{i}$ lie in $\partial U_{i}$. Orient $s_{i}$ and $l_{i}$ parallel to $k_{i}$ and orient $m_{i}$ using $k_{i}$ and the "right hand rule". To do a Dehn twist in the surface $T_{g} \times\{j\}$ along the curve $k_{i}$ we split $S^{3}$ along $T_{g} \times\{j\}$, do a full twist in one of the two annuli bounded by the copies of $k_{i}$ and $s_{i}$ and sew $S^{3}$ back together along $T_{g} \times\{j\}$.

This is equivalent to removing $U_{i}$ and sewing it back in so that a meridian is sewn to $m_{i} \pm s_{i}$ where the sign is determined by the direction of the twist. To see this, before removing $U_{i}$ just push it down a little into the surface $T_{g} \times[j-1, j]$, so that the annulus bounded by $k_{i}$ and $s_{i}$ lies in the boundary of $U_{i}$.

If the meridian of $U_{1}$ is sewn to $m_{1} \pm s_{1}$, then the meridian of $U_{2}$ is sewn to $m_{2} \mp s_{2}$ since the twists are in opposite directions. We assume (by renumbering if necessary) that the meridian of $U_{1}$ is sewn to $m_{1}+s_{1}$.

In the homology of the complement of $k_{1}$, we have $s_{1}=l_{1}+c m_{1}$ for some integer $c$ then $L\left(s_{1}, k_{1}\right)=L\left(l_{1}, k_{1}\right)+c L\left(m_{1}, k_{1}\right)$ where $L($, stands for linking number. Thus $c=L\left(s_{1}, k_{1}\right)=L\left(-s_{2}, k_{1}\right)=L\left(-k_{2}, k_{1}\right)=$ $-L\left(k_{2}, k_{1}\right)$. Similarly $s_{2}=l_{2}-L\left(k_{2}, k_{1}\right) m_{2}$.

If we do framed surgery, say on $k_{1}$, with framing $t$, then the meridian is sewn to $l_{1}+t m_{1}=s_{1}+L\left(k_{2}, k_{1}\right) m_{1}+t m_{1}$. If we choose $t=-L\left(k_{\eta}, k_{1}\right)+1$, then the Dehn surgery and the framed surgery have the same effect. By an analogous argument, the Dehn surgery at $k_{2}$ has the same effect as framed surgery at $k_{2}$ with framing $-L\left(k_{2}, k_{1}\right)-1$. Thus we have shown that every homology 3 -sphere has the asserted representation.

Now consider the manifold $M^{3}$ defined by any framed link satisfying the hypothesis of the theorem. Following Kirby [8], we 
construct a simply connected 4-manifold $W^{4}$ that $M^{3}$ bounds. The intersection matrix of $W^{4}$, with respect to the basis $\left\{f_{1}(a), f_{1}(b), \cdots\right.$, $\left.f_{n}(b)\right\}$, as in the hypothesis of the theorem, is a $2 n \times 2 n$ matrix with framing numbers on the diagonal and linking numbers off the diagonal. We can think of this matrix as an $n \times n$ matrix with $2 \times 2$ matrix entries. Off diagonal entries have the form $\left[\begin{array}{rr}x & -x \\ -x & x\end{array}\right]$ and diagonal entries have the form $\left[\begin{array}{cc}-q+1 & q \\ q & -q-1\end{array}\right]$. Using symmetric row and column operations, we see that this matrix is equivalent to a matrix with off diagonal entries $\left[\begin{array}{ll}0 & 0 \\ 0 & 0\end{array}\right]$ and diagonal entries $\left[\begin{array}{ll}0 & 1 \\ 1 & 0\end{array}\right]$ or $\left[\begin{array}{ll}1 & 1 \\ 1 & 0\end{array}\right]$. (Add the first column to the second column and then the first row to the second row to get first column off diagonal entries like $\left[\begin{array}{ll}x & 0 \\ x & 0\end{array}\right]$, first row off diagonal entries like $\left[\begin{array}{cc}x & -x \\ 0 & 0\end{array}\right]$ and $\left.] \begin{array}{cc}-q+1 & 1 \\ 1 & 0\end{array}\right]$ on the diagonal, etc.) Thus the index of this matrix and the index of $W^{4}$ is zero. Since the matrix is unimodular, $M^{3}$ is a homology sphere (see [8]).

There is the following method for computing the $\mu$-invariant of a homology 3-sphere (see [5]). Let $W^{4}$ be any simply connected 4-manifold that $M^{3}$ bounds and let $F^{2}$ be a characteristic surface. Then $\mu\left(M^{3}\right)$, as an integer $\bmod 2=1 / 8$ (index $W^{4}-F \cdot F+8$ (Kervaire invariant of $F$ )). In our case index $W^{4}=0$, and a characteristic element in $H_{2}\left(W^{4}\right)$ corresponds to the sum of the basis elements with odd framing numbers (so that $g_{j}$ is even). This can be verified by direct computation. Thus a characteristic surface $F$ can be taken as a disjoint union of tori, each torus consisting of the union of an $f_{j}(A)$ and two discs in the attached 2-handles. If $F_{j}$ is a torus component of $F$ corresponding to $f_{j}(A)$, then $F_{j} \cdot F_{j}=\left(f_{j}(a)+\right.$ $\left.f_{j}(b)\right) \cdot\left(f_{j}(a)+f_{j}((b))=f_{j}(a) \cdot f_{j}(a)+2 f_{j}(a) \cdot f_{j}(b)+f_{j}(b) \cdot f_{j}(b)=-q_{j}+\right.$ $1+2 q_{j}-q_{j}-1=0$. Thus $F \cdot F=0$. The Kervaire invariant of a component $F_{j}$ is just the $\operatorname{Arf}$ invariant of $f_{j}(A)$ as it is embedded in $S^{3}$ (see [5] for a definition of Kervaire invariant). Thus two of the three terms in the formula for $\mu\left(M^{3}\right)$ vanish and we are done.

\section{REFERENCES}

1. J. Birman, On the equivalence of Heegaard splittings of closed, orientable 3-manifolds, preprint.

2. —_, On Siegel's modular group, Math. Ann., 191 (1971), 59-68.

3 . - Poincarés conjecture and the homotopy group of a closed orientable 2-mani. fold, preprint.

4. J. Birman and H. Hilden, Heegaard splittings of branched coverings of $S^{3}$, Trans. Amer. Math. Soc., 213 (1975), 315-352.

5. S. Cappell and J. Shaneson, Some new four manifolds, Ann. of Math., (2) 104 (1976), 61-72.

6. D. Johnson, Homeomorphisms of a surface which art trivially on homology, to appear 
in the Proc. Amer. Math. Soc.

7. S. Kaplan, Lecture Notes Prepared in Connection with the Summer Institute on Algebraic and Geometric Topology held at Stanford University, Stanford, CA, August 2-21, 1976, S1-S47.

8. R. Kirby, A calculus for framed links in $S^{3}$, Invent. Math., 45 (1978), 35-56.

9. J. Powell, Two theorems on the mapping class group of surfaces, to appear in the Proc. Amer. Math. Soc.

Received December 26, 1979. This research was partially supported by an NSF grant.

University of Hawail at Manoa

Honolule, HI 96822 



\section{PACIFIC JOURNAL OF MATHEMATICS}

\section{EDITORS}

DONALD BABBITT (Managing Editor)

University of Galifornia

Los Angeles, California 90024

Hugo RossI

University of Utah

Salt Lake City, UT 84112

C. C. MOORE AND ANDREW OGG

University of California

Berkeley, CA 94720

\section{J. DugundjI}

Department of Mathematics University of Southern California

Los Angeles, California 90007

R. Finn AND J. Milgram

Stanford University

Stanford, California 94305

\section{ASSOCIATE EDITORS}

R. ARENS

E. F. BECKENBACH

B. H. NeUmanN

F. WOLF

K. YosHIDA

\section{SUPPORTING INSTITUTIONS}

UNIVERSITY OF ARIZONA

UNIVERSITY OF BRITISH COLUMBIA

CALIFORNIA INSTITUTE OF TECHNOLOGY

UNIVERSITY OF CALIFORNIA

MONTANA STATE UNIVERSITY

UNIVERSITY OF NEVADA, RENO

NEW MEXICO STATE UNIVERSITY

OREGON STATE UNIVERSITY
UNIVERSITY OF OREGON

UNIVERSITY OF SOUTHERN CALIFONIA

STANFORD UNIVERSITY

UNIVERSITY OF HAWAII

UNIVERSITY OF TOKYO

UNIVERSITY OF UTAH

WASHINGTON STATE UNIVERSITY

UNIVERSITY OF WASHINGTON 


\section{Pacific Journal of Mathematics}

\section{Vol. 94, No. 1 \\ May, 1981}

Willy Brandal, Conditions for being an FGC domain $\ldots \ldots \ldots \ldots \ldots \ldots \ldots$

Allan Calder and Frank Williams, Incompressibility of maps and the

homotopy invariance of Čech cohomology $\ldots \ldots \ldots \ldots \ldots \ldots \ldots \ldots \ldots$

Jacques Chaumat, Quelques propriétés du couple d'espaces vectoriels

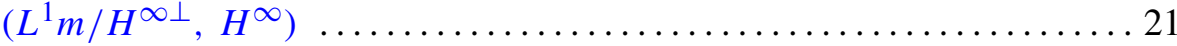

Manfred Droste and Rüdiger Göbel, Products of conjugate permutations . . 47

Jean Esterle, Rates of decrease of sequences of powers in commutative

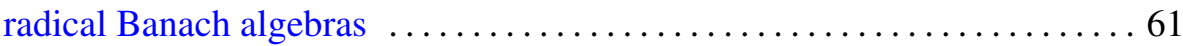

Allan Fryant, Ultraspherical expansions and pseudo analytic functions . . . 83

John Hannah, Homogenization of regular rings of bounded index. II . . . . . 107

Shigeru Haruki, On the theorem of S. Kakutani-M. Nagumo and J. L. Walsh

for the mean value property of harmonic and complex polynomials $\ldots .113$

Hugh M. Hilden, Representations of homology 3-spheres $\ldots \ldots \ldots \ldots \ldots 125$

Craig Huneke, A characterization of locally Macaulay completions . . . . . 131

Takesi Isiwata, Closed ultrafilters and realcompactness ................. 139

Joseph Weston Kitchen, Jr. and David A. Robbins, Tensor products of

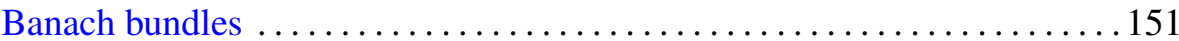

Allan J. Kroopnick, Note on bounded $L^{p}$-solutions of a generalized Liénard

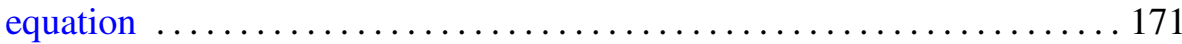

Ajay Kumar and Ajit Kaur Chilana, Spectral synthesis in products and quotients of hypergroups

Charles Livingston, Homology cobordisms of 3-manifolds, knot

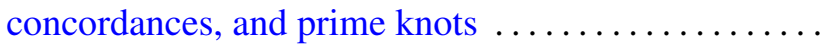

Hans Opolka, Projective representations of finite groups in cyclotomic fields

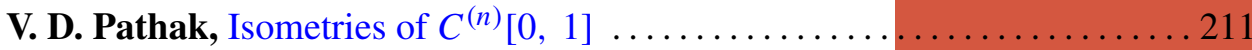

Mark Allan Pinsky, On the spectrum of Cartan-Hadamard manifolds . . . . . 2223

Judith Roitman, The number of automorphisms of an atomic Boolean

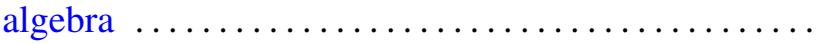

Kai Wang, Locally smooth torus group actions on integral cohomology complex projective spaces 